\title{
Research on the use of artificial neural networks for the myocardial infarction diagnosis
}

\author{
P I Katkov ${ }^{1}$, N S Davydov ${ }^{1,2}$, A G Khramov ${ }^{1,2}$ and A N Nikonorov ${ }^{1,2}$ \\ ${ }^{1}$ Samara National Research University, Moskovskoe Shosse 34A, Samara, Russia, 443086 \\ ${ }^{2}$ Image Processing Systems Institute of RAS - Branch of the FSRC "Crystallography and \\ Photonics" RAS, Molodogvardejskaya street 151, Samara, Russia, 443001
}

e-mail: katkov.p.i@gmail.com, alexander.khramov@gmail.com

\begin{abstract}
In this paper, the use of artificial neural networks for the myocardial infarction diagnosis is investigated. For the analysis, 169 ECG records were taken from the database of the Massachusetts University of Technology, of which 80 correspond to healthy patients and 89 correspond to patients who have a myocardial infarction. Each signal has been preprocessed. The result of preprocessing each signal is a common segment consisting of 1000 samples. To detect myocardial infarction, a convolutional neural network consisting of two convolutional layers was used. For accuracy of the neural network leave-one-out crossvalidation was used. The best results of the experiments are obtained with the neural network for leads V1, V2, AVF.
\end{abstract}

\section{Introduction}

Artificial neural networks are now widely used in different clinical research and its clinical use shows high quality results especially in signal analysis such as electrocardiogram (ECG) signals. Cardiovascular diseases are the main cause of health loss in most developed countries, therefore the premature detection of cardiovascular diseases is a very important issue. One of the most dangerous among cardiovascular diseases is myocardial infarction. In order to detect myocardial infarction, the doctor analyzes the results of electrocardiography.

There are many different methods for analyzing ECG signals. For example, methods based on blind signal separation [1], the multilayer method of support vectors [2], and others.

The main objective of this study is to study the applicability of artificial neural networks for the diagnosis of myocardial infarction by electrocardiogram. The main problems of every myocardial infarction detection method are complexity and a poor theoretical basis and these problems need to be solved for future developments and studies.

We compare our method of myocardial infarction detection with several others such as Blind Signal Separation (BSS) method [1]. BSS was used for the extraction of the raw signal and Naive Bayes classifier was used for myocardial infarction detection. In that study the algorithm gave $96.77 \%$ 
of accuracy, but it is hard to recreate this result using the information which is given in the paper. The second study is based on the conversion of ECG signal to 3D image and the using of multilayer support vector machine for classification [2]. However, it is also possible to reconstruct the structure of ECG signal in order to filter the signal with a risk of data loss [3]. The ECG signal represents anatomical structure of the heart, its parts and the process of working. One of the last developed method of myocardial infarction detection is based on wavelet transformation[4]. This new approach allows to do fast and accurate classifications using information from energy level of wavelet transformation.

In this paper, we proposed the new approach based on discrete wavelet transformation during preprocessing stage and convolutional neural network in the classification stage. At the first stage, there is a selection of the cardiac cycle in order to reduce the total amount of data and bring all the data to one template form. This is done with use of an algorithm that is based on a discrete wavelet transform of the original signal $[5,6,7]$.

At the second stage, the binary classification problem is solved using a convolutional neural network. This is where the architecture for the neural network is composed and its learning takes place. The patient data set was taken from the database of the Massachusetts Institute of Technology [8].

Also in this paper, to get the result of the classification close to what can be obtained by the practical application of the algorithm, a leave-one-out cross validation technique was used.

\section{Signal preprocassing}

\subsection{Segmantation}

The first step in bringing the signal to the required form is its segmentation into cardiac cycles, which will allow you to continue working not with the whole signal, but only with its part containing the most important information about the patient. Segmentation is performed using discrete wavelet transform.

\subsubsection{Trend removal and signal filtering using discrete wavelet transform}

The raw signals used in this study have different lengths and amplitudes. Each of them also has a wandering trend line, which affects the position of each cardiac cycle and can cause unwanted distortions during further processing. An example of a raw ECG signal with a wandering trend line present is shown in Figure 1.

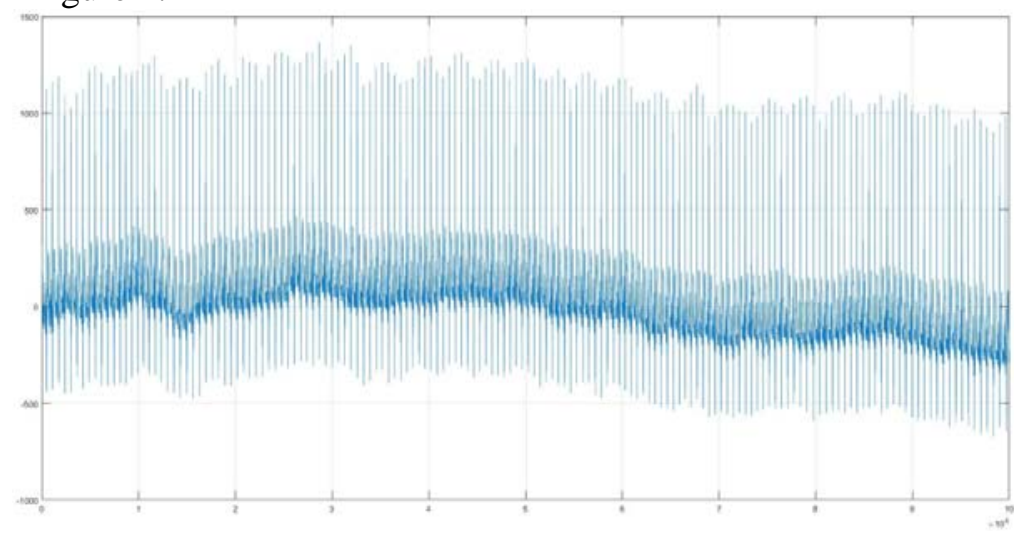

Figure 1. Raw ECG signal.

The wandering of the trend line of the ECG signal is associated with physical movements of the chest in the process of breathing the patient. This noise is low-frequency physical noise and its elimination is necessary for obtaining a stable signal and its subsequent processing.

The frequency range of the trend line is between 0 and $0.5 \mathrm{~Hz}$. The method used to remove a wandering trend line is based on decomposition using discrete wavelet transform into 8 levels and the subsequent restoration of the signal to 8 levels without using additional level coefficients. Due to the 
fact that the signal was restored from those coefficients that contain all the main details of the source signal, except for the trend line, the signal shown in Figure 2 will be received at the output of the inverse transform.

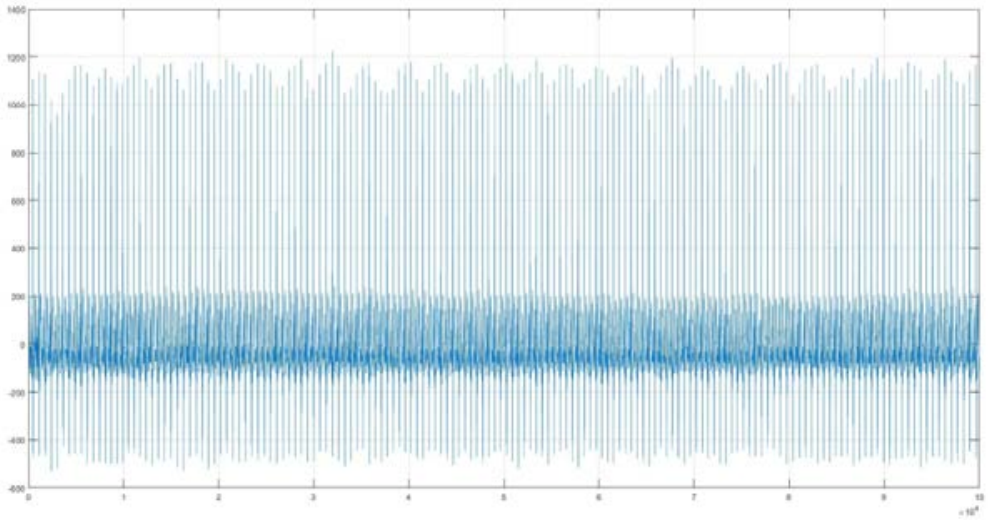

Figure 2. The raw signal without a trend.

Also, to prepare the signal for further processing, it is necessary to remove high-frequency noise. This can be done by decomposing the signal into 3 levels of discrete wavelet transform and restoring the signal according to the coefficients of the third and additional levels, thereby removing highfrequency noise from the signal.

\subsubsection{R-peak detection and signal segmentation}

To proceed the signal segmentation, it is required to select the most important point of each cardiac cycle - the R-peak. The frequency range corresponding to the QRS complex and R-peak is between 3 and $40 \mathrm{~Hz}$, which means that the signal will require decomposition into 5 levels of discrete wavelet transform and the subsequent restoration of the signal using coefficients 4 and 5 levels.

After the signal is restored, it will contain only peak values at those positions which correspond to the R-peaks in the original signal. Peaks are detected by successively searching for a maximum with a window width of half the sampling frequency and a maximum of at least $10 \%$ of the amplitude of the maximum point in the reconstructed signal. The result of projecting the found points on the original signal is shown in Figure 3.

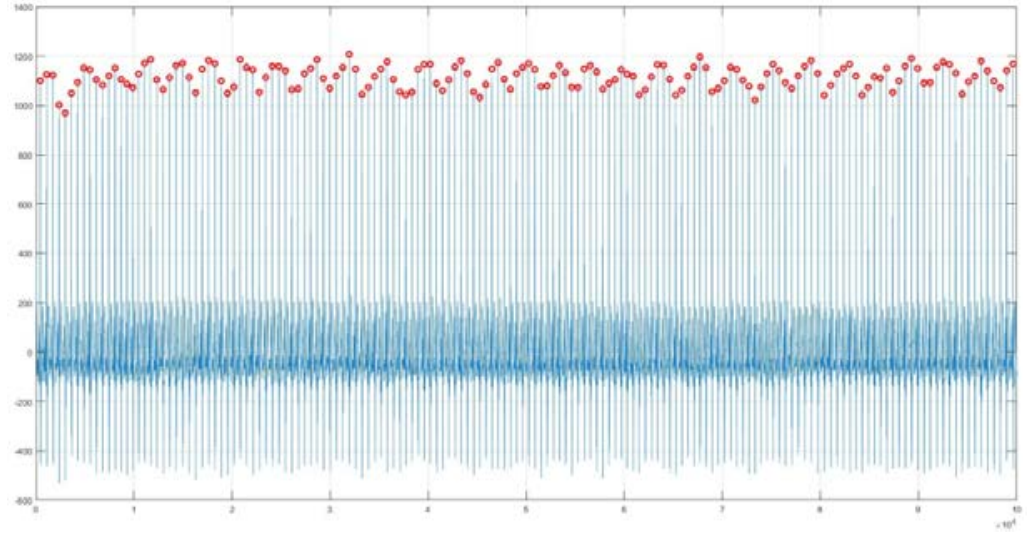

Figure 3. Detected R-peaks.

The final step of segmentation of the ECG signal will be the cutting of the signal into cardiac cycles relative to the R-peak. The length of the cardiac cycle of each patient is different and depends on his pulse and how long the electrocardiogram was taken. Therefore, the following formula was developed to calculate the length of the patient's cardiac cycle:

$$
L=\frac{\text { LengthofSignal }}{\text { Pulse }} \text {. }
$$


After calculating the length of the cardiac cycle, it is necessary to distinguish it relative to each Rpeak. To do this, take an interval equal to half of the length of the entire cycle to the left of the R-peak and an the same interval to the right of the R-peak.

Thus, the signal will be evenly divided into cardiac cycles of the same length, but for each patient this length will be different. The entire set of segments obtained as a result of dividing the ECG signal is shown in Figure 4.

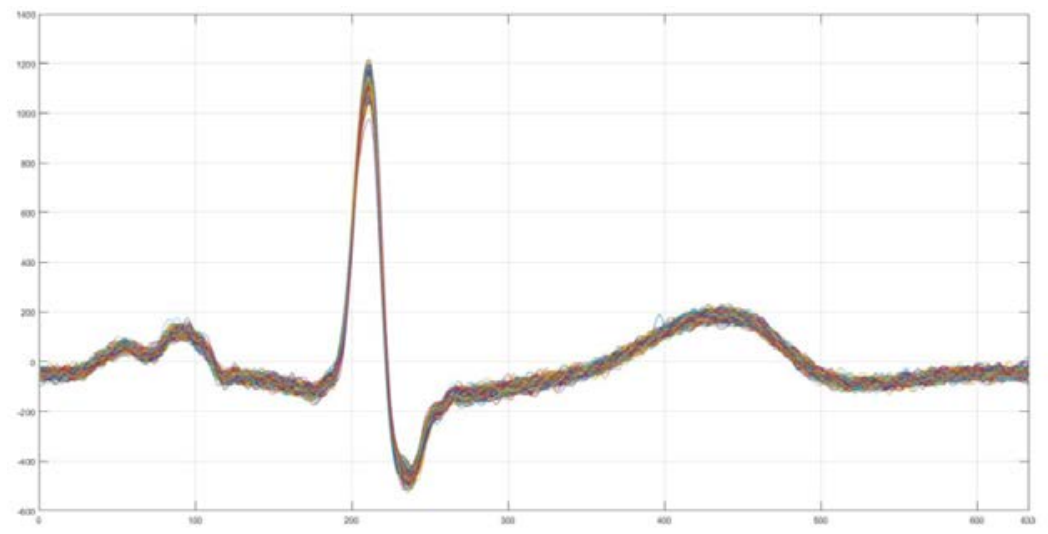

Figure 4. A set of cardiac cycles obtained from a raw ECG signal.

\subsection{Calculation of a common standard ECG signal segment}

The final step in converting the ECG signal to the common standard segment will be averaging the set of selected segments. This procedure is necessary in order to obtain the general shape of the entire set of segments and drown out low-frequency noises. It is worth mentioning that this step may drown out erroneously identified cardiac cycles that are not. For example, if in the process of ECG removal any physical noise was recorded with a frequency equal to the frequency of the QRS complex, then it can be defined as part of the heartbeat. In this regard, averaging will significantly reduce the contribution of the defective clock to the overall information segment of the signal. Also in the case of pathological signals, various heart sounds and heart damage can affect the waveform. However, the detection of damage data can be based on the selection of the shape - the average standard segment, and the subsequent finding of the greatest deviation from this segment.

The result of the final stage of processing is shown in Figure 5.

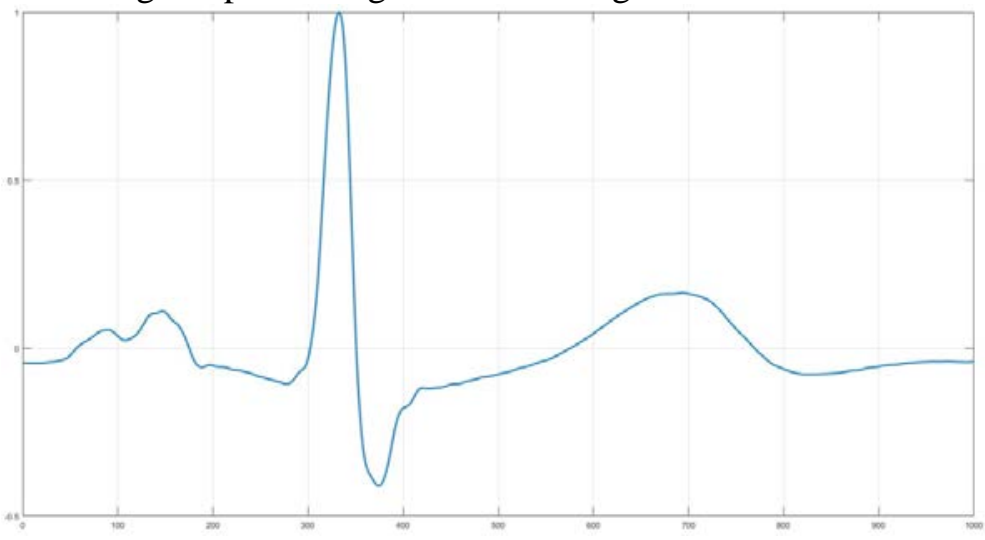

Figure 5. Common mean segment ECG signal.

\section{Architecture of the convolutional neural network}

After preprocessing signals for all 169 input records, we obtain an array of dimension $(169,600, \mathrm{~N})$, where $\mathrm{N}$ is the number of leads taken. This array will be fed to the input of our neural network along with the array, which contains the answers to each entry (1, if the patient is healthy, 0 , if he has a posterodiaphragmatic myocardial infarction). We implement the neural network using the Keras library in the Python language.

The following figure 6 shows the architecture of the convolutional neural network. 


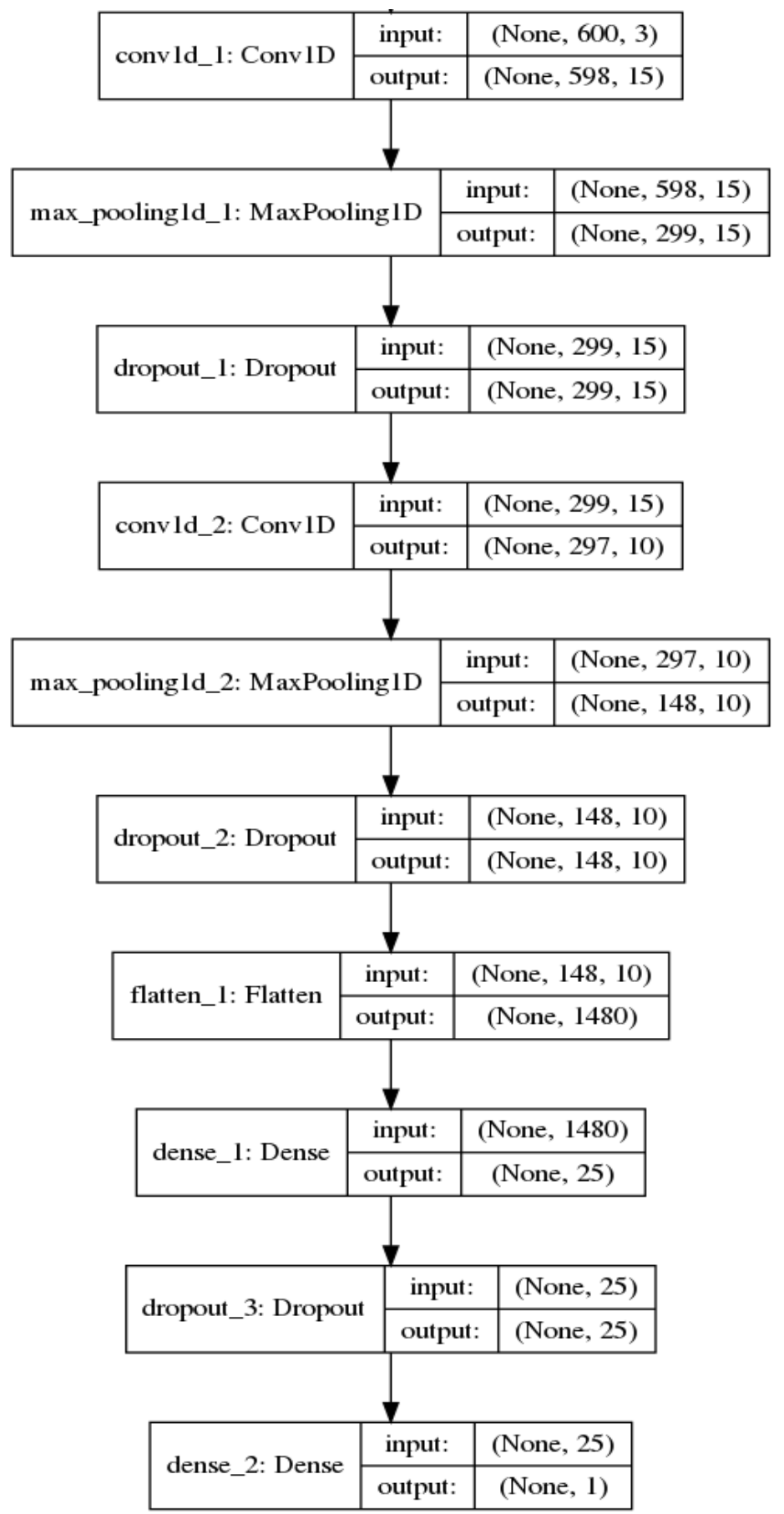

Figure 6. Architecture of the convolutional neural network.

A convolutional layer is a set of feature maps. The number of attribute cards is determined experimentally. If you take a large number of cards, the recognition quality will increase, but the computational complexity will increase too. Two convolutional layers were chosen because, with a smaller choice of layers, the accuracy of the network becomes noticeably worse, and with a larger choice of layers, it is not noticeably better. Each convolutional layer is 15 and 10 feature maps, respectively. To reduce the dimensions of the maps of the previous layer, we use the subsample operation MaxPooling. In order to prevent retraining of the neural network on each convolutional layer, the Dropout method is used. The fully connected part of the network includes two levels. All weights were chosen experimentally. 
The network is trained using the error back-propagation algorithm and is optimized in accordance with the Adam optimization algorithm.

The Adam method converts a gradient as follows:

$$
\begin{gathered}
\mathrm{S}_{\mathrm{t}}=\alpha \cdot \mathrm{S}_{\mathrm{t}-1}+(1-\alpha) \cdot \nabla \mathrm{E}_{\mathrm{t}}^{2} ; \mathrm{S}_{0}=0 ; \\
\mathrm{D}_{\mathrm{t}}=\beta \cdot \mathrm{D}_{\mathrm{t}-1}+(1-\beta) \cdot \nabla \mathrm{E}_{\mathrm{t}} ; \mathrm{D}_{0}=0 ; \\
g_{t}=\frac{D_{t}}{1-\beta} \cdot\left(\frac{1-\alpha}{S_{t}}\right)^{1 / 2} ; \\
\Delta \mathrm{W}_{\mathrm{t}}=\eta \cdot\left(\mathrm{g}_{\mathrm{t}}+\rho \cdot \mathrm{W}_{\mathrm{t}-1}\right)+\mu \cdot \Delta \mathrm{W}_{\mathrm{t}-1},
\end{gathered}
$$

where $\eta$ - learning rate, $\nabla \mathrm{E}_{\mathrm{t}}$ - loss function gradient, $\mu$ - moment ratio, $\Delta \mathrm{W}_{\mathrm{t}-1}$-weights changing in the previous iteration, $\rho$ - regularization coefficient, $\mathrm{W}_{\mathrm{t}-1}$ - weights on the previous iteration, $\alpha$ $=0.999, \beta=0.9$.

Since we are solving a binary classification problem, the logistic error function was chosen as the error function, which looks like this:

$$
E(w)=-\sum_{i=1}^{N}\left(y_{i} \ln a_{i}+\left(1-y_{i}\right) \ln \left(1-a_{i}\right)\right) \rightarrow \min ,
$$

where $y$ - target vector, $a$ - output vector.

\section{Results}

For an experimental study, a program was launched for a data set consisting of 169 records with different numbers of leads. Pre-processing was performed for each input signal. In order to test how successfully our model is able to work in practice, element-by-element cross-validation was performed. In this case, a separate observation is used as a test set of data, and the remaining observations from the initial set are used as a training one. The cycle repeats until each observation is used once as a test. Table 1 shows the learning results of the neural network depending on the leads taken.

Table 1. The results of the neural network, depending on the different leads.

\begin{tabular}{cccc}
\hline Leads & Sensitivity & Specificity & Accuracy \\
\hline II, V1, V2, AVF & 0.8315 & 0.7375 & 0.7870 \\
\hline II, III, AVF & 0.7416 & 0.6500 & 0.6982 \\
\hline V1, V2 & 0.8876 & 0.7625 & 0.8284 \\
\hline II, III, V1, V2, AVF & 0.7640 & 0.8250 & 0.7929 \\
\hline AVF & 0.8315 & 0.8000 & 0.8166 \\
\hline V1, V2, AVF & $\mathbf{0 . 9 2 1 3}$ & $\mathbf{0 . 8 5 0 0}$ & $\mathbf{0 . 8 8 7 6}$
\end{tabular}

After launching the program with different input leads, the neural network showed the best results when the input data are leads V1, V2, AVF.

Compare the results with other methods of detecting myocardial infarction. It should be noted that in different articles different methods are used to extract signs from the ECG signal, and in some of them the entire signal is analyzed, and therefore a direct comparison of the classification result with each of them is not completely correct. Also, the difference between the presented feature extraction algorithm can be that the algorithm considers three ECG leads of twelve.

The first algorithm considered for comparison is based on quantization of the learning vector [6]. LVQ can be understood as a special case of an artificial neural network. LVQ-network has 2 layers: competing and linear. The competing layer performs clustering of vectors, and the linear layer relates clusters to the target classes specified by the user. Before giving a signal to the input of the neural network, it is pre-processed and extracted from it the cardiac cycle. However, the article does not explain how many leads were taken to analyze the results of the electrocardiogram, and there is no accuracy value in that paper.

The following algorithm is based on the blind signal separation method [1]. The problem of blind signal separation (BSS) and noise is an extremely relevant and complex mathematical problem, but its 
solution is non-trivial and the material of the article does not explain how this problem was solved and whether the data was cross-validated. Algorithms based on the support vector method have different directions. In one of the considered articles, the ECG signal is converted to a three-dimensional view and the calculation of three-dimensional signs of the disease and the subsequent application of the multi-layer support vector machine (MSVM) [2].

The results of the comparison of various approaches to the detection of myocardial infarction are shown in Table 2.

Table 2. Comparison with other methods.

\begin{tabular}{cccc}
\hline Methods & Sensitivity & Specificity & Accuracy \\
\hline This study & 0.9213 & 0.8500 & 0.8876 \\
\hline LVQ & 0.8700 & 0.8300 & - \\
\hline BSS & 0.9837 & 0.9404 & 0.9677 \\
\hline MSVM & 0.8682 & 0.9105 & 0.8543
\end{tabular}

\section{Conclusion}

In this paper, the possibility of using convolutional neural networks to determine myocardial infarction was demonstrated. A preliminary selection of data and its preparation for training was done. A convolutional neural network consisting of two convolutional layers was constructed and trained. An experimental study was conducted, which showed that the neural network shows the best results when the input data are leads V1, V2, AVF. On these leads, the neural network showed results: accuracy 0.8876 , sensitivity -0.9213 , specificity -0.8500 . The classification was carried out using the elementwise cross-validation method to obtain a result that most adequately shows the operation of the algorithm in practical application.

\section{References}

[1] Devika M G and Aneesh R P 2016 Myocardial infarction fetection using hybrid BSS method International Conference on Communication Systems and Networks 1167

[2] Dhawan A, Wenzel B, George S, Gussak I, Bojovic B and Panescu D 2012 Detection of acute myocardial infarction from serial ecg using multilayer support vector machine 34th Annual International Conference of the IEEE EMBS 12704

[3] Smelkina N A, Kosarev R N, Nikonorov A V, Bairikov I M, Ryabov K N, Avdeev A V and Kazanskiy N L 2017 Reconstruction of anatomical structures using statistical shape modeling Computer Optics 41(6) 897-904 DOI 10.18287/2412-6179-2017-41-6-897-904

[4] Swain S, Patra D 2018 Multiscale energy based suitable wavelet selection for detection of myocardial infarction in ECG 1 Healthcare Technology Letters 61

[5] Davydov N S, Khramov A G 2018 Myocardial infarction detection using wavelet analysis of ECG signal CEUR Workshop Proceedings 2212 31-37

[6] Haddadi R, Abdelmounim E and Hanine M 2014 Discrete wavelet transform based algorithm for recognition of qrs complexes World of Computer Science and Information Technology Journal 4127

[7] Chandel S and Singh K 2016 ECG denoising using wavelet transform and soft thresholding International Journal of Advanced Research in Computer Science and Software Engineering 6370

[8] The PTB Diagnostic ECG Database 2016 URL: https://physionet.org/physiobank/ database/ptbdb/ (09.03.2019)

[9] Loginov D S 2011 Methods and tools for computer processing of electrocardiogram for the diagnosis of myocardial infarction: Dis. Cand. tech. Sciences: 05.11 .17 (Penza) p 134

\section{Acknowledgments}

With support of RFBR grants (projects 19-29-01235-mk, № 18-07-01390-A), and the state assignment of the IPSI RAS - a branch of the Federal Scientific-Research Center "Crystallography and Photonics" of the RAS (agreement № 007-ГЗ/Ч3363/26). 